\title{
Incidence of Childhood Cancers at a Tertiary Hospital in Kenya: 2009 - 2019
}

\section{Mutua Irene* and Mwika Peter}

University of Nairobi, Kenya

*Corresponding Author: Mutua Irene, University of Nairobi, Kenya.

DOI: $10.31080 /$ ASPE.2020.03.0272
Received: June 16, 2020

Published: July 25, 2020

(C) All rights are reserved by Mutua Irene and

Mwika Peter.

\begin{abstract}
Objective: To assess the prevalence and incidence rate of Leukemia, Retinoblastoma, Wilms tumour, Lymphoma, Malignant neoplasm of connective and soft tissue and Neuroblastoma at Kenyatta National Hospital (KNH) and to determine the age at diagnosis, gender and mortality rate of above cancer types.

Design: It was a retrospective descriptive study conducted at KNH using health records from childhood cancer registry of children aged 0 - 12 years who were diagnosed or referred to Kenyatta National Hospital with cancer from the period January 2009 to December 2019. Based on patient's inpatient number, we excluded from our analyses all re-hospitalizations of the same patients avoiding duplications.

Results: The prevalence of Leukemia from our study was 30.6\% (n-650), retinoblastoma 23.3\% (n-495), Wilms tumour 16.9\% (n359), Lymphoma 16\% (n-339), Malignant neoplasm of connective and soft tissue 9.1\% (n-193) and Neuroblastoma at 4.1\% (n-87). Leukemia had the highest incidence rate of 70.6 per million while Neuroblastoma had the lowest at 9.4 per million children between age 0 - 12 years. All these cancer types occurred more common between age 0 - 4 years at $64 \%$ (n-1359). Males were mostly affected than females. Retinoblastoma had the lowest mortality rate at $6.9 \%$ while leukemia and Neuroblastoma had the highest mortality rate, both at $47.1 \%$.

Conclusion: Leukaemia, Retinoblastoma, Wilms tumour and Lymphoma are the most prevalent type of cancers at KNH, in that order. With this data, we hope that it will aid policy decisions with an aim of improving patient care quality.

Keywords: Cancer Registry; Childhood Tumours; Incidence; Mortality Rate; Prevalence
\end{abstract}

\section{Introduction}

Childhood cancers has been on the rise globally being diagnosed in 385,509 cases yearly in children aged 0 - 19 years worldwide [1]. The current incidence of childhood cancer is unknown at Kenyatta National Hospital (KNH) which is the national referral hospital in Nairobi, Kenya and the largest in East and Central Africa. Majority of the children with cancer therefore undergo treatment at KNH. In this particular paper, we focussed on six common types of cancers in children. These include Leukemia, Lymphoma,
Retinoblastoma, Nephroblastoma, Neuroblastoma and Malignant neoplasm of connective and soft tissue. Despite different multidisciplinary teams existing at our facility consisting of paediatric oncologists, paediatric radiologists, ophthalmologist, haematologists, paediatric surgeons, pathologists and child psychologists, we still have some mortalities. Childhood cancers according to World Health Organization in low- and middle-income countries (LMICs) have only a $20 \%$ cure rate of cancers compared to high income countries with $80 \%$ cure rate of childhood cancers [2]. 


\section{Aim of the Study}

The aim of this paper is to raise awareness of the incidence of these conditions at our facility. This will go along to improve patient management through the multidisciplinary teams that already exists and influence decision making by the policy makers in the quality of care of childhood cancers in Kenya.

\section{Methods}

Design

Retrospective descriptive study.

\section{Setting and population}

Childhood Cancer registry and statistics unit at the health records and information department at KNH. Children aged $0-12$ years who were diagnosed or referred to Kenyatta National Hospital with cancer from the period January 2009 to December 2019.

\section{Broad objective}

To assess the incidence of childhood cancers at Kenyatta National Hospital.

\section{Specific objectives}

- To determine the cancer prevalence and incidence rate of leukemia, lymphoma, retinoblastoma, connective and soft tissue malignant neoplasm, neuroblastoma and nephroblastoma at $\mathrm{KNH}$.

- $\quad$ To determine age at diagnosis and gender of patients with leukemia, lymphoma, retinoblastoma, connective and soft tissue malignant neoplasm, neuroblastoma and nephroblastoma at KNH.

- To assess cancer mortality rate of leukemia, lymphoma, retinoblastoma, connective and soft tissue malignant neoplasm, neuroblastoma and nephroblastoma at KNH.

Data collection

Using ICD 10 coding, we selected the particular cancer types from the data compiled from the Childhood Cancer registry in the statistics unit at KNH. This will include, the patients number, gender, age at time of diagnosis and type of cancer diagnosed and whether the patient is alive or dead. Based on patient's inpatient number, we excluded from our analyses all re-hospitalizations of the same patients avoiding duplications.

\section{Data analysis}

Data collected from cancer registry in the statistics unit at KNH was exported to STATA 14 software for cleaning and analyses. The final data set after dropping all duplicates included 2123 children aged 0 - 12 years who met the inclusion criteria. Descriptive statistics such as frequencies and percentages are used for categorical variables. Prevalence was determined by dividing the number of cancer cases by type to the total cancer cases in the study expressed as a percentage. The cancer incidence rates were calculated by dividing the number of cancer cases by type divided by the total population of children aged $0-12$ years in Nairobi County multiplied by a million.

Results

\section{Cancer prevalence rate at $\mathrm{KNH}$}

From the table below, the most prevalent types of cancers cases at $\mathrm{KNH}$ includes: Leukaemia (30.6\%), Retinoblastoma (23.3\%), Wilms tumour (16.9\%), Lymphomas (16\%), malignant neoplasm, connective and soft tissue (9.1\%) and Neuroblastoma (4.1\%). Acute lymphoblastic and Acute myeloblastic remains the most prevalent types of leukaemia while Hodgkin and Non-Hodgkin remains the most prevalent lymphomas. The summary is presented in table 1 below.

\begin{tabular}{|l|c|c|}
\hline Disease & N & Prevalence \\
\hline Total Leukaemia & 650 & $30.6 \%$ \\
\hline Acute lymphoblastic leukaemia [ALL] & 414 & $19.5 \%$ \\
\hline Acute myeloblastic leukaemia [AML] & 140 & $6.6 \%$ \\
\hline $\begin{array}{l}\text { Chronic lymphocytic leukaemia of B-cell } \\
\text { type }\end{array}$ & 30 & $1.4 \%$ \\
\hline $\begin{array}{l}\text { Chronic myeloid leukaemia [CML], BCR/ } \\
\text { ABL-positive }\end{array}$ & 26 & $1.2 \%$ \\
\hline Others (C94;C95;C95.9) & 40 & $1.9 \%$ \\
\hline Retinoblastoma & 495 & $23.3 \%$ \\
\hline Wilms tumour & 359 & $16.9 \%$ \\
\hline Total Lymphomas & 339 & $16.0 \%$ \\
\hline Hodgkin lymphoma & 88 & $4.1 \%$ \\
\hline Non-Hodgkin lymphoma & 134 & $6.3 \%$ \\
\hline Burkitt lymphoma & 84 & $4.0 \%$ \\
\hline Others (C82.2,9; C83.2,3,4,5,9; C84.5,5) & 33 & $1.6 \%$ \\
\hline $\begin{array}{l}\text { Malignant neoplasm, connective and soft } \\
\text { tissue (C49.0-C49.9) }\end{array}$ & 193 & $9.1 \%$ \\
\hline Neuroblastoma & 87 & $4.1 \%$ \\
\hline Total & 2123 & $100 \%$ \\
\hline
\end{tabular}

Table 1: The cancer prevalence rate of leukemia, lymphoma, retinoblastoma, connective and soft tissue malignant neoplasm, neuroblastoma and nephroblastoma.

C94.0- Acute erythroid leukemia; C95.0- Acute leukemia of unspecified cell type; C95.9- Leukemia unspecified.

C82.2- Follicular lymphoma; C83.2- Mixed small and large cell (diffuse); C83.3- Diffuse Large B cell lymphoma; C83.4- Immunoblastic (diffuse); C83.5- Lymphoblastic (diffuse) lymphoma; C83.9- Nonfollicular (diffuse) Non- Hodgkin lymphoma, unspecified; C84.5Other Mature T/NK- cell lymphoma. 
Age at diagnosis of various types of cancer at $\mathrm{KNH}$

The study found that a greater percentage of cancer cases are diagnosed between 0 - 4 years. The study found that $88.1 \%$ of retinoblastoma, $80.2 \%$ of wilms tumour, $79.3 \%$ of neuroblastoma and $56 \%$ of malignant neoplasm of connective and soft tissue are diagnosed between 0 to 4 years of age. However, more than $50 \%$ of leu- kaemia cases and $60 \%$ of Lymphoma are diagnosed between 5 and 12 years of age. The summary of the prevalence and the incidence rates is presented in table 2 and the average population of children 0 - 12 years in Nairobi county in Kenya according to the Kenya National Bureau of Statistics (KNBS) is presented in table 3 below.

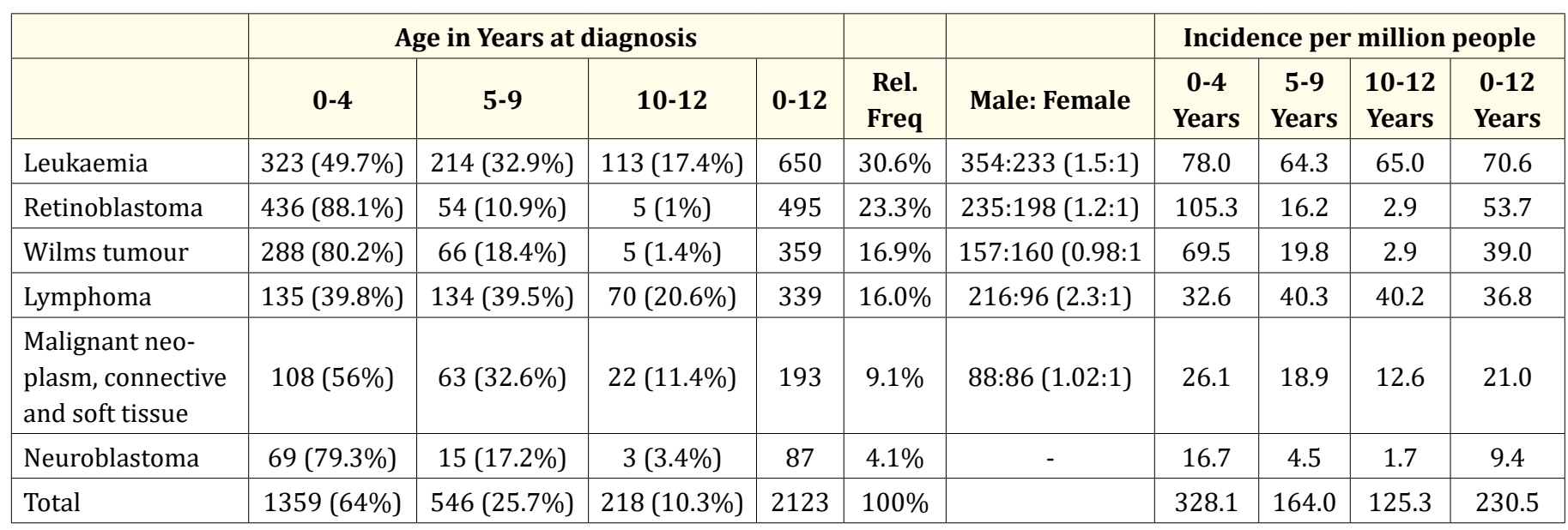

Table 2: Age at diagnosis of leukemia, lymphoma, retinoblastoma, malignant neoplasm of connective and soft tissue, neuroblastoma and nephroblastoma.

\begin{tabular}{|l|c|c|c|}
\hline \multicolumn{4}{|c|}{ Nairobi county } \\
\hline Years & Population 2009 & Population 2019 & Average \\
\hline $0-4$ & 395466 & 524,987 & 460227 \\
\hline $5-9$ & 307,013 & 432,712 & 369863 \\
\hline $10-12$ & 154,262 & 232,306 & 193284 \\
\hline \multicolumn{4}{|l|}{ Source: KNBS National Census 2009 and 2019 } \\
\hline
\end{tabular}

Table 3: Average population of children 0-12 years Nairobi county.

\section{Cancer mortality rates at $\mathrm{KNH}$}

The study established that Leukaemia and Neuroblastoma cases had the highest mortality rates at $47.1 \%$ respectively, followed by Malignant neoplasm of connective and soft tissue (34.7\%), Wilms tumour (33.7\%) and Lymphoma (32.4\%). Retinoblastoma had the lowest mortality rates at $6.9 \%$. The summary is presented in figure 1 and summarised in table 4 below.

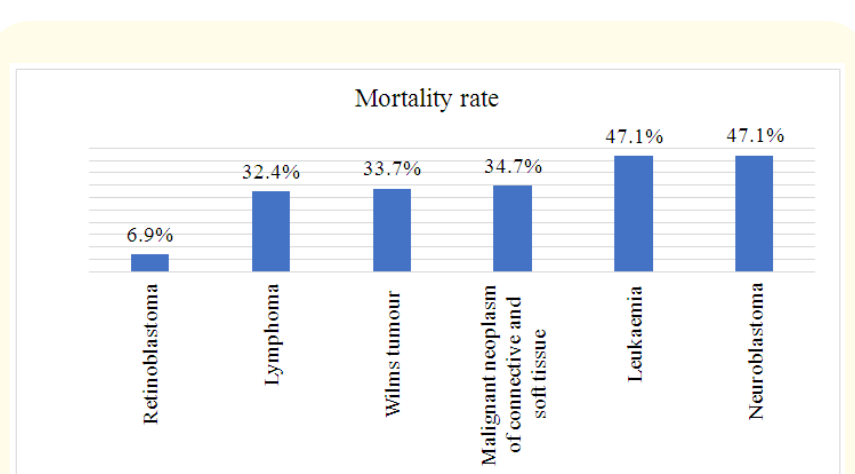

Figure 1: Cancer mortality rate of leukemia, lymphoma, retinoblastoma, connective and soft tissue malignant neoplasm, neuroblastoma and nephroblastoma at $\mathrm{KNH}$. 


\begin{tabular}{|l|c|c|c|c|}
\hline & $\begin{array}{c}\text { Mortality } \\
\text { rates }\end{array}$ & Alive & Dead & Total \\
\hline Leukaemia & $47.10 \%$ & 344 & 306 & 650 \\
\hline Neuroblastoma & $47.10 \%$ & 46 & 41 & 87 \\
\hline $\begin{array}{l}\text { Malignant neoplasm of } \\
\text { connective and soft tissue }\end{array}$ & $34.70 \%$ & 126 & 67 & 193 \\
\hline Wilms tumour & $33.70 \%$ & 238 & 121 & 359 \\
\hline Lymphoma & $32.40 \%$ & 229 & 110 & 339 \\
\hline Retinoblastoma & $6.90 \%$ & 461 & 34 & 495 \\
\hline
\end{tabular}

Table 4: Number of death, alive and mortality rates of various cancer cases.

\section{Discussion}

Leukemia is a malignant disorder of white blood cells caused by abnormal differentiation of haemopoietic stem cells and progenitor cells in the bone marrow. This leads to accumulation of immature cells in the bone marrow suppressing other blood cells causing bone marrow failure. Patients thus present with neutropenia, anaemia and thrombocytopenia. The prevalence of leukemia from our study was $30.6 \%$ (n- 650) having increased from $21.3 \%$ [3] and $20.8 \%$ [ 4 ] in our setup. It was $10.2 \%$ in another study conducted in Nigeria [5]. It was the commonest childhood cancer from our study with acute lymphoblastic type being $19.5 \%$ (n-414) followed by acute myeloblastic leukemia at $6.6 \%(n-140)$. This is almost similar to a study done in Western Kenya where acute lymphoblastic leukemia was $15 \%$ [6]. The male to female ratio was 1.5: 1 from our study. Leukemia was diagnosed in children at age 0 - 4years in $49.7 \%$, between age 5 - 9 years in $32.9 \%$ and between age $10-12$ years in $17.4 \%$. According to international incidence of childhood cancer, leukemia was more common at $36.1 \%$ in children aged 0 - 4 years and it was the leading cancer in all regions [1]. The incidence per million people for leukemia between 0 - 4 years was 78 children, between age 5 - 9 years was 64 children and between age 10 12 years was 65 children. Cumulative incidence between age 0 - 12 years was 71 per a million children. In Japan, the age standardized incidence rate ( 0 - 14yrs) was 42 while in England, was 46.8 per a million children [7] lower than in our study.

Retinoblastoma is malignant tumour of the retina which occurs due to mutations occurring in a retinal cell. The prevalence of retinoblastoma was $23.3 \%$ (n-495) in our study. It is the second most common childhood cancer in our setup. This has increased from 8.5\% [4]. In a study conducted in Tanzania, the prevalence was

$12.9 \%$ [8] while a study in Malawi had a prevalence of $11.3 \%$ [9]. In a study conducted in Nigeria, the prevalence was 21\% [5] almost similar to our study. The male to female ratio was 1.2: 1 from our study. Retinoblastoma was diagnosed in children at age 0 - 4 years in $88.1 \%$, between age 5 - 9 years in $10.9 \%$ and between age $10-12$ years in $1 \%$. The incidence per million people for retinoblastoma between 0 - 4 years was 105 children, between age 5 - 9 years was 16 children and between age 10 - 12 years was 3 children. Cumulative incidence between age 0 - 12 years was 54 per a million children. In Japan, the age standardized incidence rate ( 0 - 14yrs) was 4.6 while in England, was 4.5 per a million children [7] hence more common in our setup.

Wilms tumour also known as nephroblastoma is malignant tumour of the kidney. The prevalence of Wilms tumour was $16.9 \%$ (n-359) in our study. It was the third most common childhood cancer from our study.

In a study by Mwanda in the same setup, nephroblastoma had a prevalence of $14 \%$ [10] having increased slightly compared to other studies which had lower prevalence at 8.5\% [3,4] and 3.4\% [11] in the same setup. In a study by Mgaya in Tanzania, the prevalence was $6.7 \%$ [8] while in another study it was $6.6 \%$ [5]. The male to female ratio was 0.98: 1 from our study. Wilms tumour was diagnosed in children at age $0-4$ years in $80.2 \%$, between age 5 - 9 years in $18.4 \%$ and between age 10 - 12 years in $1.4 \%$. The incidence per million people for Wilms tumour between 0 - 4 years was 69 children, between age 5 - 9 years was 20 children and between age 10 - 12 years was 3 children. Cumulative incidence between age 0 - 12 years was 39 per a million children. In Japan, the age standardized incidence rate $(0-14 \mathrm{yrs})$ was 3.5 while in England, was 8.9 per a million children [7] which is much lower compared to our study.

Lymphoma is a lymphoproliferative disorder involving lymph nodes, bone marrow, spleen, gastrointestinal tract or lungs. The prevalence of lymphoma was $16.0 \%$ (n-339) being the fourth most common childhood cancer in our study having decreased from $17.5 \%$ from a study conducted in the same setup [4]. In another study, the prevalence was $22.4 \%$ [5]. Non- Hodgkin lymphoma was the commonest at $6.3 \%$, Hodgkin lymphoma at $4.1 \%$ and Burkitt's lymphoma at $4.0 \%$ from our study. In a study by Christina in the same setup, Hodgkin lymphoma was the commonest at $6.9 \%$ followed by Non- Hodgkin lymphoma at $4.3 \%$ then Burkitt's lym- 
phoma at 3.3\% [4]. From Kijabe Hospital pathology database in Kenya, Non- Hodgkin lymphoma was $8.5 \%$. Hodgkin lymphoma at $7.6 \%$ and Burkitt's lymphoma at $16.6 \%$ [11]. In a study conducted in Tanzania, Non- Hodgkin lymphoma was 6.6\% [8] while in Malawi, the prevalence of Hodgkin's lymphoma was 4.8\% [9] almost similar to our study. The male to female ratio was 2.3: 1 from our study. Lymphoma was diagnosed in children from our study at age 0 - 4 years in $39.8 \%$, between age 5 - 9 years in $39.5 \%$ and between age 10 - 12 years in $20.6 \%$. The incidence per million people for lymphoma between 0 - 4 years was 33 children, between age 5 - 9 years was 40 children and between age 10 - 12 years was 40 children. Cumulative incidence between age 0 - 12 years was 37 per a million children. In Japan, the age standardized incidence rate 0 - 14yrs) was 11.1 while in England, was 14.1 per a million children [7] which is lower compared to our study.

Malignant neoplasm of connective and soft tissue arises from embryonal mesenchyme. It can affect the head, face and neck, thorax, abdomen, pelvis, upper limb including the shoulder and lower limbs including the hip. It had a prevalence of $9.1 \%$ from our study. A study conducted in Nigeria had a prevalence of $14.9 \%$ [5]. Cristina., et al. found a prevalence of $13.9 \%$ [4], 5.2\% [3] in the same setup in Kenya, while it was 3.5\% in another study in Kijabe Hospital in Kenya [11]. In a study by Mgaya in Tanzania, the prevalence was $6.7 \%$ [8] while in Malawi, the prevalence was $4.3 \%$ [9]. The male to female ratio was 1.02:1 from our study. This tumour was diagnosed in children at age 0 - 4 years in $56 \%$, between age 5 - 9 years in $32.6 \%$ and between age 10 - 12 years in $11.4 \%$. The incidence per million people for malignant neoplasm of connective and soft tissue between 0 - 4 years was 26 children, between age 5 - 9 years was 19 children and between age 10 - 12 years was 13 children. Cumulative incidence between age 0 - 12 years was 21 per a million children. In Japan, the age standardized incidence rate (0 - 14yrs) was 6.6 while in England, was 8.8 per a million children [7] which is lower compared to our study.

Neuroblastoma arises from fetal neural crest cells that form the sympathetic nervous system. The prevalence of neuroblastoma was $4.1 \%$ in our study. It was the least common childhood cancer from our study. This has increased from $1.7 \%$ in a study conducted in the same setup [4] while it was $2.6 \%$ in a study conducted in Nigeria [5]. Neuroblastoma was diagnosed in children at age $0-4$ years in $79.3 \%$, between age 5 - 9 years in $17.2 \%$ and between age 10 - 12 years in $3.4 \%$. The incidence per million people for neuro- blastoma between 0 - 4 years was 17 children, between age 5 - 9 years was 4 children and between age 10 - 12 years was 2 children. Cumulative incidence between age 0 - 12 years was 9 per a million children. We had a lower incidence compared to Japan whose age standardized incidence rate (0 - 14yrs) was 16.2 while in England, it was 9.6 per a million children [7].

Over a 10year period (2009 - 2019) from our study, the mortality rate of retinoblastoma was at $6.9 \%$, lymphoma at $32.4 \%$, Wilms tumour at $33.7 \%$, malignant neoplasm of connective and soft tissue at $34.7 \%$, leukemia was $47.1 \%$ and neuroblastoma was at $47.1 \%$. A study performed in Rwanda had a mortality rate of Wilms tumour at $30.2 \%$ [12] slightly lower in comparison to our study. Mortality occurs in LMICs due to obstacles in assessing care such as financial reasons which lead to treatment abandonment as well, lack of adequate human resource and equipment like the radiotherapy machine, lack of diagnosis, misdiagnosis or diagnosis delay [2]. Cancer mortality can be either disease related or treatment related. Disease related deaths may be due to late presentation hence advanced disease with metastasis as seen in our setup or due to relapse on therapy. Treatment related deaths include haematological toxicity causing neutropenia [12].

\section{Conclusion}

Leukemia was the commonest childhood cancer in our setup followed by retinoblastoma then Wilms tumour. With this data, we hope that it will aid policy decisions in Kenya with an aim of improving patient care quality.

\section{Funding}

No funding or grant support.

\section{Authorship}

All authors attest that they meet the current ICMJE criteria for Authorship.

\section{Conflict of Interest}

The following authors have no financial disclosures: (MI, MP).

\section{Bibliography}

1. Eva SF and Murielle C LAG. "International incidence of childhood cancer, 2001-10: a population- based registry study". The Lancet Oncology 18.6 (2017): 719-731. 
2. World Health Organization. Cancer in children: key facts. Fact Sheets (2018).

3. Macharia WM. "Childhood cancers in a referral hospital in Kenya: a review”. East African Medical Journal 73.10 (1996).

4. Stefan C., et al. "Cancer of childhood in sub-Saharan Africa". Ecancer 11 (2017).

5. Babatunde T., et al. "Pattern of childhood cancer in University College Hospital, Ibadan during 1991-2010 and comparison with the previous three decades". Paediatrics and International Child Health 35.2 (2015): 144-150.

6. Mostert S and Njuguna F KL. "Epidemiology of diagnosed childhood cancer in Western Kenya". Archives of Disease in Childhood 97.6 (2012): 508-512.

7. Kayo N and Yuri I WM. "Childhood cancer incidence and survival in Japan and England: A population- based study (19932010)". Cancer Science 109 (2018): 422-434.

8. Mgaya EM KJN. "Histopathology of malignant tumours of childhood in Tanzania". East African Medical Journal 77.8 (2000): 435-439.

9. Mukiibi JM Banda L LNG. "Spectrum of childhood cancers in Malawi 1985-1993". East African Medical Journal 72.1 (1995): 25-29.

10. Mwanda OW. "Cancers in children younger than 16 years in Kenya”. East African Medical Journal 76.1 (1999): 3-9.

11. Margaron FC and Poenaru D NA. "Pediatric Cancer spectrum in Kenya: a histopathologic review". Pediatric Surgery International 26.8 (2010): 789-794.

12. Cyprien S and Mary JX SNE. "Treating nephroblastoma in Rwanda: Using International Society of Pediatric Oncology Guidelines in a novel oncologic care Model". Journal of Global Oncology 2.3 (2016): 105-13.

\section{Assets from publication with us}

- Prompt Acknowledgement after receiving the article

- Thorough Double blinded peer review

- Rapid Publication

- Issue of Publication Certificate

- High visibility of your Published work

Website: www.actascientific.com/

Submit Article: www.actascientific.com/submission.php

Email us: editor@actascientific.com

Contact us: +919182824667 